\title{
Molecular Diagnostics in the Public Health Laboratories
}

\section{DEBORAH JOSKO}

\section{LEARNING OBJECTIVES}

1. Discuss various molecular viral assays performed at the public health laboratory.

2. Describe West Nile virus surveillance protocols.

3. Explain Qiagen QIAmp BioRobot 9604 methodology.

4. Compare and contrast Applied Biosystems $7900 \mathrm{HT}$ and 7500 Fast real-time PCR systems.

5. Describe protocols utilized to identify rabies.

6. Discuss the principle of PFGE in the identification of enteric organisms.

7. State the principle of the Bactec MGIT 960 Mycobacterial Detection System.

8. List select and non-select agents identified in the bioterrorism and molecular detection services laboratory.

ABBREVIATIONS: $\mathrm{AFB}=$ acid-fast bacilli; $\mathrm{CDC}=$ Centers for Disease Control and Prevention; CSF = cerebrospinal fluid; DFA = direct fluorescent antibody; DNA = deoxyribonucleic acid; ELISA = enzyme-linked immunosorbent assay; FITC = fluorescein isothiocyanate; GIS = Geographical Information System; H1N1 = influenza A virus; LRN = Laboratory Response Network; MDS = Molecular Detection Services; MGIT = mycobacteria growth indicator tube; $\mathrm{MTB}=$ Mycobacterium tuberculosis; $\mathrm{PCR}=$ polymerase chain reaction; PFGE = pulsed field gel electrophoresis; RNA = ribonucleic acid; STEC = shiga-toxin producing Escherichia coli; $\mathrm{TB}=$ tuberculosis; $\mathrm{WHO}=$ World Health Organization; WNV = West Nile virus

INDEX TERMS: Type A influenza, West Nile virus, rabies, pulsed field gel electrophoresis, real-time PCR, Mycobacteria growth indicator tube, Mycobacterium tuberculosis, multiplex PCR, direct fluorescent antibody, bioterrorism
Clin Lab Sci 2010;23(4)242

Deborah Josko, PhD, M(ASCP) ${ }^{C M}, M L T, S M$, University of Medicine and Dentistry of New Jersey, Scotch Plains, NJ.

Address for correspondence: Deborah Josko, PhD, $M(A S C P)^{C M}, M L T, S M$, associate professor, Department of Clinical Laboratory Sciences, Medical Laboratory Science Program, University of Medicine and Dentistry of New Jersey, 1776 Raritan Road, Scotch Plains, NJ 07076. (908)889-2422. joskotda@umdnj.edu.

The author does not endorse any company or product and has no financial gain or otherwise interest in the products presented.

\section{ACKNOWLEDGEMENTS}

The author would like to acknowledge the following individuals at the New Jersey Department of Health and Senior Services for their collaborative support in the development of this article: Donna Bartron, supervising laboratory technician; Patricia Bryant, molecular virologist, supervising medical technologist; Nelson Delgado, PhD, research scientist 1 microbiology; Gordon Fratz, supervisor, rabies laboratory, microbiologist 2; Thomas Kirn, MD, PhD, associate medical director, Public Health Laboratory Services; Shawn Lee, microbiologist 5; Paul Seitz, program manager bacteriology, research scientist 1 microbiology; Martha Smith, director, policy, planning and regulatory compliance; and Bruce Wolf, program manager virology, research scientist 1 microbiology.

This article will give a brief overview of some of the molecular methodologies and assays performed at the New Jersey State Department of Health and Senior 


\section{FOCUS: MOLECULAR METHODS IN CLINICAL MICROBIOLOGY}

Services laboratory in Trenton, NJ. Assays to be discussed include influenza A, swine flu, West Nile virus, rabies, Salmonella and Shigella, Mycobacterium tuberculosis, and Streptococcus pneumoniae. Methodologies such as real-time PCR, pulsed field gel electrophoresis (PFGE) and multiplex PCR will also be discussed.

\section{MOLECULAR VIRAL ASSAYS}

\section{Introduction}

Samples for viral testing are sent to the public health laboratory for identification and confirmation. If a medical laboratory scientist identifies an unsubtypable strain of type A influenza virus, they send the isolate to the public health laboratory for confirmation. If the public health lab confirms the type A unsubtypable influenza strain, an influenza 2009 A (H1N1)pdm² (formerly "swine flu") confirmation test is ordered. ${ }^{1}$ Screening, routine flu surveillance, and confirmation testing are done using real-time polymerase chain reaction (PCR), however when confirming, a different set of probes and primers is used in the master mix than those used for screening and surveillance.

Human specimens for suspected West Nile virus (WNV) are sent to the public health laboratory since most clinical laboratories do not test for WNV or do not have the volume to justify keeping identification kits on hand. Specimens go to the public health serology department where enzyme immunoassay testing is performed on serum. Specimens such as mosquitoes and birds are analyzed using real-time PCR. ${ }^{1}$ ELISA is the method of choice for human specimens since the virus is typically no longer present in the blood once symptoms occur. ${ }^{1}$ In addition, local health officials send samples to the public health laboratory to monitor mosquito populations carrying the WNV and to test avian specimens suspected of being infected with WNV. Mosquitoes are captured at specific geographical locations using Geographical Information System (GIS) coordinates, where they are speciated and sent to the laboratory for testing. ${ }^{1}$ Local health officials will only spray areas with insecticide in regions where mosquito pools test positive for WNV. Routine surveillance protocols are in place and are part of the normal routine in mosquito pool monitoring during the summer months.

\section{Molecular Viral Testing Instrumentation}

The virology laboratory is equipped with robotic isolation instruments and multiple real-time PCR thermocyclers. Sample preparation for the isolation of viral nucleic acids is performed on the Qiagen QIAmp BioRobot 9604. ${ }^{3}$ This instrument, used in conjunction with the appropriate kit, extracts and purifies DNA or RNA using robotic technology. Once reagents and buffers are prepared and placed in the system, the specimens - whether serum, plasma, CSF, cell free body fluids, or cell culture supernatants $s^{3,4}$ are added to the individual wells where purification and extraction is performed. This instrument is not fully automated since the plates must be removed and centrifuged to remove any excess ethanol used during the procedure. ${ }^{3,4}$ Once the nucleic acids are purified, they are transferred to either 96-well or 384-well plates containing the master mix (which was manually prepared) and placed in the thermocycler where the samples are subjected to realtime PCR. ${ }^{1}$

The laboratory uses AB Applied Biosystems 7900HT and 7500 Fast Real-Time PCR Systems. The 7900HT system can accommodate 96 and 384 well formats while the 7500 accommodates only 96., Both systems can generate rapid results; $30+$ minutes in rapid mode for both systems - 96 wells; 52 minutes in rapid mode for 384 wells (7900HT); and less than 2 hours for both systems in standard mode ${ }^{5,6}$ CDC protocols recommend use of standard mode on all assays. ${ }^{1,2}$ Once real-time PCR is complete, the results are downloaded, interpreted, and reported.

The virology laboratory uses robotics and real-time PCR in a number of assays. Table 1 lists the various viral pathogens tested in the laboratory. For assays listed, nucleic acid extraction can be performed either using robotic technology or manual techniques. Mosquito pools are best analyzed by adding diluent, homogenizing the sample, then manually extracting the RNA since varying volumes are obtained. ${ }^{1}$ This ensures standardization of sample preparation on all specimens received. Routine flu surveillance on the other hand relies on robotic extraction due to the volume of samples received during flu season. ${ }^{1}$ All assays listed utilize real-time PCR for detection. 


\section{FOCUS: MOLECULAR METHODS IN CLINICAL MICROBIOLOGY}

Table 1. Viral Pathogens Tested at the New Jersey Department of Health and Senior Services Laboratory. ${ }^{1}$

Influenza A and B virus
Influenza subtypes $\mathrm{H} 1$ and $\mathrm{H} 3$
Swine flu (Influenza 2009 A (H1N1) pdm)
West Nile virus
West Nile virus surveillance
Norovirus
Mumps
Eastern equine virus
St. Louis encephalitis
La Crosse encephalitis
Orthopox viruses
Nonorthopox viruses

\section{RABIES LABORATORY}

Although rabies is a preventable viral infection if prompt medical care is initiated, more than 55,000 individuals die each year (95\% of deaths occur in Africa and Asia). ${ }^{7,8}$ The majority of human cases are caused by the bite of an infected dog, therefore it is important to keep up to date with rabies vaccinations for dogs as well as other indoor pets such as cats and ferrets.,

According to the World Health Organization (WHO), immediate cleansing at the site of the bite and immunizations (human rabies immune globulin and three-injection series of rabies vaccine) can prevent the onset of rabies symptoms (fever, headache, fatigue, respiratory, gastrointestinal and/or central nervous system involvement) in $100 \%$ of exposures. ${ }^{8,9}$

Human deaths caused by exposure to rabid animals such as wild foxes, raccoons, skunks, jackals, and wolves are relatively rare. Humans most likely become infected with rabies by contact with an infected dog or cat. ${ }^{8}$ In cases of bites, it is important to determine whether the animal in question is infected with the rabies virus as soon as possible. If at all possible, the animal should be captured, humanely sacrificed, and its head should be sent to a laboratory facility that performs rabies testing. Whether the animal is captured or not, post-exposure prophylaxis should be administered immediately.

New Jersey's State Department of Health and Senior Services facility has an active rabies laboratory where they may receive over 600 samples per month. ${ }^{1}$ The heads of animals, whether bats, cats, dogs, squirrels, or raccoons, are sent for diagnostic testing. Whole bodies are accepted as long as the animal is no larger than a squirrel. ${ }^{1}$ Veterinarians, animal control officers, local health officials, or the public at large collect the samples, which are then brought to designated drop off points and transported by a courier service to the public health laboratory for rabies testing. Once the brain arrives in the laboratory, the supervisor extracts the cerebellum and brain stem and processes the sample using an established protocol. ${ }^{1}$ The sample is fixed to several slides then analyzed using a direct fluorescent antibody (DFA) assay. Positive and negative controls are run with each batch. The CDC recommends using antirabies conjugates from two different sources, preferably two different monoclonal antibody pools. ${ }^{10}$ This will reduce the risk of non-recognition of any one variant. ${ }^{10}$ Fluorescein isothiocyanate (FITC) conjugated antirabies antibodies are added directly to the slide and bind the rabies antigen if present. ${ }^{10}$ Unbound material is washed away and once the slides are dried, they are cover slipped and placed under the fluorescent microscope for review. Figure 1 illustrates a microscopic image of both a positive and negative DFA result for rabies using animal nervous tissue. ${ }^{11}$ The public health rabies laboratory provides 24 hour turn around times for results performed Monday through Friday.

\section{MOLECULAR BACTERIOLOGY ASSAYS}

One of the most widely recognized tools available for investigative epidemiology purposes is pulsed field gel electrophoresis (PFGE). ${ }^{9}$ First described in 1984 by Schwartz and Cantor, this technique, which separates large DNA fragments, produces distinct DNA fingerprint patterns that are used not only for epidemiology purposes, but for strain typing and identification techniques. ${ }^{9,12}$ Other applications for PFGE in the field of molecular biology are numerous and diverse. ${ }^{13}$

At the New Jersey Department of Health and Senior Services laboratory there are two labs for testing enteric isolates. Laboratory scientists in the enterics laboratory perform confirmatory testing on an isolate utilizing automated identification systems, serotyping, flagellar stains, and antisera. Once the isolate is confirmed as either a Salmonella, Shigella, E. coli 0157 or non 0157 


\section{FOCUS: MOLECULAR METHODS IN CLINICAL MICROBIOLOGY}

shiga-toxin producing E. coli (STEC), the isolate is then processed in the PFGE laboratory, which ultimately generates a DNA fingerprint pattern of the isolate. ${ }^{1}$ PFGE techniques involve many steps, summarized here.

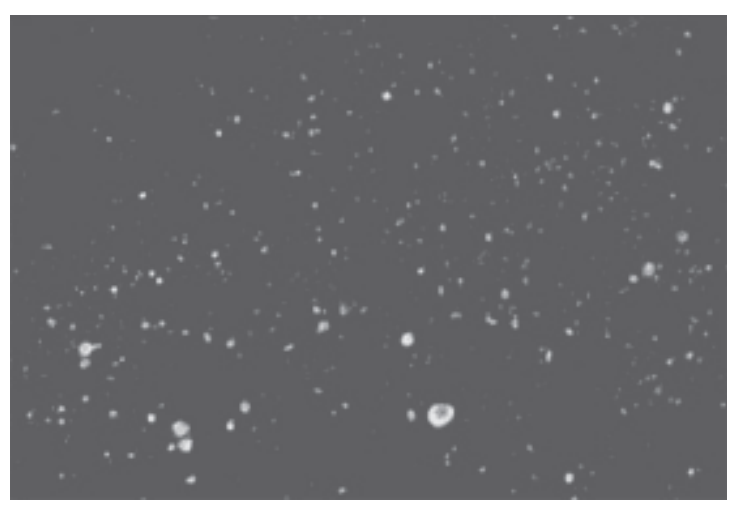

Positive DFA

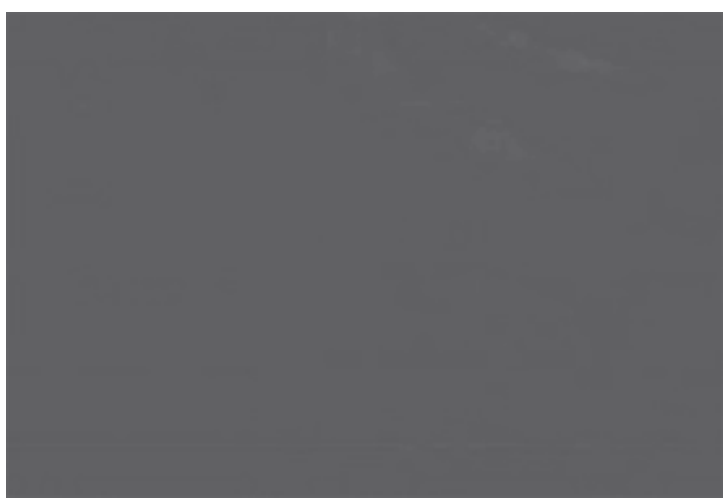

Negative DFA

Figure 1. Microscopic Image of a Positive and Negative DFA Test for Rabies. Image courtesy of the Centers for Disease Control and Prevention (CDC). ${ }^{11}$ The illustration on the top represents a positive rabies DFA of brain tissue using fluorescent microscopy. The image on the bottom is negative.

DNA is initially implanted in agarose plugs, then treated with enzymes to destroy protein and lipid material leaving just the nuclear material for analysis. ${ }^{1,12,13,14}$ After several washes with buffer, the plug is cut into approximately $2 \mathrm{~mm}$ pieces, subjected to specific restriction enzymes, and loaded onto a comb. ${ }^{1,14}$ After the gel is poured over the comb, the comb is removed, buffer is added and an electrical current is applied to the apparatus. ${ }^{1,13,14}$ The current changes orientation throughout the process as it bombards the agarose gel at various angles. This differs from conventional electrophoresis where the current is unidirectional and constant. This is done to "force" the large fragments of DNA through the gel. ${ }^{1,9,13,14}$ The current is applied for approximately 18 hours before the process is complete. ${ }^{1,14}$ The gel is then stained with ethidium bromide and examined under ultraviolet light. ${ }^{1}$ A digital image is taken of the DNA fingerprint pattern and uploaded to New Jersey and the CDC's databases, where they are compared to recorded patterns for commonalities. ${ }^{1}$ The computer software has the ability to run comparison studies on each pattern generated. New Jersey isolates are compared state-wide, whereas the CDC looks at patterns on a national level. ${ }^{1}$ The information obtained is made available to the state's epidemiology division for further analysis.

\section{MYCOBACTERIOLOGY}

The New Jersey Department of Health and Senior Services laboratory receives a large number of sputum samples from various county clinics and TB clinics throughout the state. ${ }^{1}$ The bulk of the workload is surveillance, i.e. follow up on confirmed TB cases in order to determine whether the organism has been eliminated and/or whether the patient has been compliant with their TB medications. ${ }^{1}$ Susceptibility testing is also done to determine if drug resistance has occurred.

Sputum samples are plated on AFB media-one solid medium and one liquid medium called the mycobacteria growth indicator tube (MGIT). The facility utilizes the automated Bactec MGIT 960 Mycobacterial Detection System. ${ }^{15}$ The MGIT, which contains $7 \mathrm{~mL}$ of modified Middlebrook $7 \mathrm{H} 9$ broth base and an oxygen-quenched fluorochrome, is more efficient in detection time and susceptibility testing compared to solid media. ${ }^{16}$ The principle behind the broth is the fluorochrome is suppressed in the presence of oxygen however, once the free oxygen is utilized by the organism and carbon dioxide is generated, the fluorochrome is released and detected under ultraviolet light. ${ }^{16}$ The instrument detects the fluorochrome signal and flags the tube as positive.

If the MGIT turns positive, a DNA probe for MTB complex can be applied directly on the broth providing enough organisms are present. If not, the MGIT is subcultured to AFB media where the DNA probe is 


\section{FOCUS: MOLECULAR METHODS IN CLINICAL MICROBIOLOGY}

performed on the colonies, once isolated. The MTB complex includes Mycobacterium tuberculosis, Mycobacterium bovis (including bacillus Calmette-Guerin strain), Mycobacterium africanum, Mycobacterium canettii, and Mycobacterium micoti. ${ }^{9}$ Once a positive is confirmed for TB complex, it is sent for susceptibility testing.

Susceptibility testing is done on the MGIT instrument where first-line tuberculosis drugs (rifampin, streptomycin, ethambutol, and isoniazid) are tested. ${ }^{1,15,16}$ If any resistance is noted, the drugs are tested again using higher concentrations. ${ }^{1}$ If resistance is still noted, the samples are sent to an outside institution for second-line drug testing.

Due to the emergence of multi-drug resistant strains of MTB, it is important the state department of health monitors patient compliance and keeps abreast of any new resistant strains. This is accomplished by surveillance programs and follow-up protocols that are part of the day-to-day operations at the facility.

\section{SELECT AND NON-SELECT AGENTS}

The New Jersey Department of Health and Senior Services provides a state of the art bioterrorism laboratory and molecular detection services (MDS) laboratory for rapid identification and detection of select and non-select agents, respectively. ${ }^{1,17}$ The bioterrorism laboratory, which is part of the Laboratory Response Network (LRN), is secure. ${ }^{17}$ It is a biosafety level 3 facility where real-time PCR is performed on select bioagents as determined by the CDC. ${ }^{1,18}$ In PCR positive cases, confirmatory testing such as DFA or DNA probes are done.

The MDS laboratory utilizes the same molecular technology as the bioterrorism laboratory (real-time PCR, DNA probes and DFA). Non-select agents such as Bordetella pertussis, Streptococcus pneumoniae, and Legionella pneumophilia are analyzed, where various serotypes of each species are identified. ${ }^{1}$ For example, laboratorians can determine Streptococcus pneumoniae serotypes by performing multiplex PCR assays using multiple sets of oligonucleotides in one master mix. The PCR reaction is a home brew assay developed by the CDC (end-point PCR) where amplicons are loaded on a gel, stained with ethidium bromide, and compared to a standard ladder to determine the unknown serotype. ${ }^{19}$ At least 91 serotypes of $S$. pneumoniae have been identified. $^{20}$ The multiplex PCR assay used at this facility can detect up to 40 of the most common serotypes. ${ }^{1}$ This information is important in seroepidemiology studies and in determining whether pneumococcal vaccines are effective against the serotypes identified. ${ }^{1,19}$

\section{SUMMARY}

At the New Jersey Department of Health and Senior Services laboratory, clinical laboratory scientists receive specimens from various health care facilities and clinics in the state, and identify and confirm organisms of particular health concern in a rapid and timely manner. Most of the assays performed are specialized tests that are not done routinely in the clinical laboratories. Identifying and monitoring infectious agents such as West Nile virus, Influenza 2009 A (H1N1)pdm, Mycobacterium tuberculosis, enteric organisms, etc. is part of the daily workload in the Public Health Laboratories. In addition, surveillance programs are in place to control the spread of various infectious agents and to aid in investigative epidemiologic purposes. The volume of samples and workload demands can be overwhelming especially in peak months such as summer (WNV) or winter (Influenza A) therefore, it was necessary to implement molecular diagnostics and robotic technology to aid in high throughput analysis. Molecular assays have become the "norm" not only in the public health laboratories but in most clinical microbiology laboratories as well. The use of molecular diagnostics has proven advantageous resulting in accurate results, decreased turn around times, and overall better patient care and outcomes.

\section{REFERENCES}

1. New Jersey Department of Health and Senior Servicespersonal communication. 2010 January 12.

2. CDC Online Newsroom. Available from http://www.cdc.gov/ media/pressrel/2010/r100622.htm. Accessed 2010 July 7.

3. QIAamp Virus BioRobot 9604 Kit. Available from http://www.qiagen.com/products/genomicdnastabilizationpurif ication/qiaampsystem/qiaampdnavirusbiorobot9604kit.aspx. Accesssed 2010 June 23.

4. QIAamp Virus BioRobot 9604 Kit Handbook. Available from http://www.qiagen.com/products/genomicdnastabilizationpurif 


\section{FOCUS: MOLECULAR METHODS IN CLINICAL MICROBIOLOGY}

ication/qiaampsystem/qiaampdnavirusbiorobot9604kit.aspx\#T $a b s=t 2$. Accessed 2010 June 23.

5. AB Applied Biosystems: 7500 Fast Real-Time PCR. Available from https://products.appliedbiosystems.com/ab/en/US/adirect /ab? cmd=catNavigate2\&catID $=603784 \& \mathrm{tab}=$ TechSpec. Accessed 2010 June 23.

6. AB Applied Biosystems: 7900 HT Fast Real-Time PCR System. Available from https://products.appliedbiosystems. com/ab/en/US/adirect/ab;jsessionid=RfpZMv6Q7p1vk944cW ShmqSVf7KpV2Z5W8YpkClVYksj72TmnWfZ!1314982431? $\mathrm{cmd}=$ catNavigate2 \&catID $=601255 \& \mathrm{tab}=\mathrm{TechSpec}$. Accessed 2010 June 23.

7. Rabies-Centers for Disease Control and Prevention. Available from http://www.cdc.gov/rabies/prevention/people.html. Accessed 2010 July 7.

8. Rabies-World Health Organization. Available from http://www.who.int/mediacentre/factsheets/fs099/en/. Accessed 2010 July 7.

9. Mahon CR, Lehman DC, Manuselis G, editors. Textbook of Diagnostic Microbiology, $4^{\text {th }}$ ed. Missouri: Saunders, Elsevier; 2010.

10. Protocol for postmortem diagnosis of rabies in animals by direct fluorescent antibody testing. Available from http://www.cdc.gov/rabies/pdf/RabiesDFASPv2.pdf. Accessed 2010 July 7.

11. CDC-Rabies Direct Fluorescent Antibody Test. Available from http://www.cdc.gov/rabies/diagnosis/direct_fluorescent_ antibody.html. Accessed 2010 July 7.
12. Schwartz DC, Cantor, CR. Separation of yeast chromosomesized DNAs by pulsed field gradient gel electrophoresis. Cell 1984;37:67-75.

13. Pulsed field electrophoresis for separation of large DNA. Available from http://www.nal.usda.gov/pgdic/Probe/v2n3/ puls.html. Accessed 2010 July 8.

14. Wickert B. Pulsed field gel electrophoresis explanation. Available from http://www.nphl.org/documents/pfgeinservice_ 000.ppt. Accessed 2010 July 8.

15. BACTEC $^{\mathrm{TM}}$ MGIT $^{\mathrm{TM}} 960$ Mycobacterial Detection System. Available from http://www.bd.com/ds/productCenter/MTBactecMgit960.asp. Accessed 2010 July 8.

16. MGIT Procedure Manual. Available from http://www.finddiagnostics.org/export/sites/default/resourcecentre/find_documentation/pdfs/mgit_manual_nov_2007.pdf. Accessed 2010 July 8.

17. Public Health and Environmental Laboratories. Available from http://www.state.nj.us/health/phel/phelorg.shtml. Accessed 2010 July 8.

18. Bioterrorism Agents/Diseases. Available from http:// emergency.cdc.gov/agent/agentlist-category.asp. Accessed 2010 July 12 .

19. Pai R, Gertz RE, Beall B. Sequential multiplex PCR approach for determining capsular serotypes of Streptococcus pneumoniae isolates. J Clin Micro 2006; 44: 124-131.

20. Adegbola R, Hill P, Secka O, and others. Serotype and antimicrobial susceptibility patterns of isolates of Streptococcus pneumoniae causing invasive disease in the Gambia 1996-2003. Trop Med Int Health. 2006;11: 1128-1135.

$\checkmark$ ASCLS wants to THANK YOU for renewing your
membership for the 2010-2011 year!
$\checkmark$ ASCLS is offering 6 online quizzes at no charge to
help with your CE needs.
$\checkmark$ For details* about this offer and order form, go
to www.ascls.org/education/memberthankyou .
$\checkmark$ We know you have choices as to which
organization you belong and we are thrilled you
chose ASCLS!
*For PF1, PF2, \& FYP members who renewed by 9/30/10
*Must complete quizzes by 7/31/11

